\title{
Dynamics of the solar chromosphere
}

\section{High-frequency modulation in ultraviolet image sequences from TRACE}

\author{
A. G. de Wijn ${ }^{1}$, R. J. Rutten ${ }^{1,2}$, and T. D. Tarbell ${ }^{3}$ \\ 1 Sterrekundig Instituut, Utrecht University, Postbus 80 000, 3508 TA Utrecht, The Netherlands \\ e-mail: [A.G.deWijn; R.J.Rutten]@astro.uu.nl \\ 2 Institute of Theoretical Astrophysics, Oslo University, PO Box 1029 Blindern, 0315 Oslo, Norway \\ 3 Lockheed Martin Solar and Astrophysics Lab, Dept. ADBS, Building 252, 3251 Hanover Street, Palo Alto, CA 94304, USA \\ e-mail: tarbell@lmsal.com
}

Received 24 July 2004 / Accepted 12 October 2004

\begin{abstract}
We search for signatures of high-frequency oscillations in the upper solar photosphere and low chromosphere in the context of acoustic heating of outer stellar atmospheres. We use ultraviolet image sequences of a quiet center-disk area from the Transition Region and Coronal Explorer (TRACE) mission which were taken with strict cadence regularity. The latter permits more reliable high-frequency diagnosis than in earlier work. Spatial Fourier power maps, spatially averaged coherence and phase-difference spectra, and spatio-temporal $\left(k_{\mathrm{h}}, f\right)$ decompositions all contain high-frequency features that at first sight seem of considerable intrinsic interest but actually are more likely to represent artifacts of different nature. Spatially averaged phase difference measurement provides the most sensitive diagnostic and indicates the presence of acoustic modulation up to $f \approx 20 \mathrm{mHz}$ (periods down to $50 \mathrm{~s}$ ) in internetwork areas.
\end{abstract}

Key words. Sun: photosphere - Sun: chromosphere - Sun: oscillations

\section{Introduction}

In this paper we continue studies of solar atmosphere oscillations based on analyzing the temporal brightness modulation in image sequences taken with the Transition Region and Coronal Explorer (TRACE) in ultraviolet passbands which sample the upper solar photosphere and low solar chromosphere. We again exploit the absence of seeing in TRACE data (apart from spaceweather particle hits) to provide extensive Fourier diagnostics for quiet-sun network and internetwork areas with excellent sampling statistics.

In Krijger et al. (2001, henceforth Paper I), these techniques were used in a comprehensive overview of quiet-sun brightness oscillation properties derived from TRACE image sequences in its three ultraviolet passbands centered at $\lambda=1700,1600$, and $1550 \AA$. In standard models of the solar atmosphere such as FALC of Fontenla et al. (1993) these passbands sample layers just below, at, and just above the temperature minimum, respectively. The subsequent paper by Rutten \& Krijger (2003) compared low-frequency ultraviolet brightness modulation at these wavelengths to the underlying white-light patterns in quiet-sun areas. Muglach (2003) analyzed TRACE ultraviolet brightness modulation maps containing an active region.

In this paper we return to the high-frequency aspects of ultraviolet brightness modulation. The data used in Paper I suffered from irregular timing intervals between successive images, severely reducing the high-frequency information content. The sequences used here have strict sampling regularity and are therefore better suited to search for high-frequency oscillation signatures. We also employ a much improved alignment method.

The obvious motivation for such searches is given by the long quest for acoustic heating of outer stellar atmospheres started by Biermann (1948) and Schwarzschild (1948). It is concisely summarized by Wunnenberg et al. (2002), to whom we refer for further background. Wunnenberg et al. (2002) employed image sequences from the Göttingen Fabry-Perot spectrometer at the German Vacuum Tower Telescope on Tenerife, scanning the non-magnetic Fe I 5434 A line which samples layers around $h=500 \mathrm{~km}$ above the white-light surface. They inferred the presence of sufficient power with $50-100$ s periodicity $(10-20 \mathrm{mHz}$ in frequency) to compensate the radiative losses of the chromosphere, with apparent spatial power concentration above intergranular lanes. In this analysis we use TRACE data to search for corroborative evidence in ultraviolet brightness modulation from the same layers.

\section{Observations and data reduction}

The TRACE mission is described by Handy et al. (1999). We use ultraviolet image sequences downloaded from the TRACE 
archive $^{1}$. They were recorded on June 1, 2003 at the request of M. Carlsson (Oslo), who suggested strict cadence regularity and low data compression in order to minimize high-frequency artifacts, in particular those arising from timing irregularities as analyzed in Sect. 5 of Paper I. TRACE was programmed to obtain such image sequences in its $1600-\AA$ and $1700-\AA ̊$ ultraviolet passbands from 8:14 to 18:34 UT. We selected uninterrupted subsequences of 1120 images starting at 11:23:12 UT and ending at 15:25:56 UT. They have strictly regular cadence at $13 \mathrm{~s}$ sampling interval in both passbands. The corresponding Nyquist frequency is $f_{\mathrm{Ny}}=38.46 \mathrm{mHz}$; the frequency resolution is $\Delta f=34.34 \mu \mathrm{Hz}$. The images sample a quiet area of $256^{\prime \prime} \times 192^{\prime \prime}$ centered at $X=-2.78^{\prime \prime}, Y=13.46^{\prime \prime}$ near the center of the solar disk, corresponding to a field of view of $512 \times 384$ square $0.5^{\prime \prime}$ pixels. The $1600-\AA ̊$ and $1700-\AA$ images were alternately exposed for 1.724 and $4.872 \mathrm{~s}$, respectively. The mid-exposure delay between the closest pairs of 1600- $\AA$ and $1700-\AA ̊$ images is $5.574 \mathrm{~s}$.

The image sequences were processed with the SolarSoft routine trace_prep described in the TRACE Analysis Guide $^{2}$. It corrects missing pixels (of which there were none in these data), replaces saturated pixels with values above 4095 , subtracts the dark field, and corrects for the flat field. The dark and flat fields were recorded on November 8, 2001 and March 11, 2003, respectively. The image brightness was normalized by the exposure time.

In the course of this analysis it became clear that precise image alignment, including corrections for differential solar rotation and for spacecraft pointing jitter, is crucial to Fourier phase-difference analysis at high frequencies, and that we should considerably improve on the method used in Paper I. In that paper, co-aligned subfields of the $1700-\AA ̊$ sequences were cross-aligned to the corresponding subfields in the $1600-\AA$ sequences. This procedure copies alignment errors from one sequence to the other and so introduces a high-frequency phase-difference signal at the retardation set by the timing offset between the exposures at the two wavelengths. It emerges in Fig. 18 of Paper I as a high-frequency drift of the spatially averaged phase-difference curves towards the average offset caused by non-simultaneous sampling shown in the center panel of Fig. 28 of Paper I. The slower cadence of the October 14, 1998 data also analyzed in Paper I caused correspondingly larger offset (Fig. 26).

In the present analysis such erroneous cross-alignment signals are reduced by significantly improving the alignment procedure. In order to minimize the use of interpolation, we measured pointing displacements per image through an elaborate procedure detailed below and then used these displacements to resample the original images directly onto an aligned grid.

We began by shifting every row of each image in solar $X$ to correct solar rotation including its differential shear, using the expression of Howard et al. (1990). We then aligned each image of 40-image 1600-Å sub-sequences to the last one of the previous set, comparably to the procedure in Paper I. Each $1700-\AA$ image was subsequently cross-aligned to the

\footnotetext{
${ }^{1}$ http://vestige. Imsal. com/TRACE

${ }^{2}$ http://moat. nascom. nasa.gov/ bentley/guides/tag
}

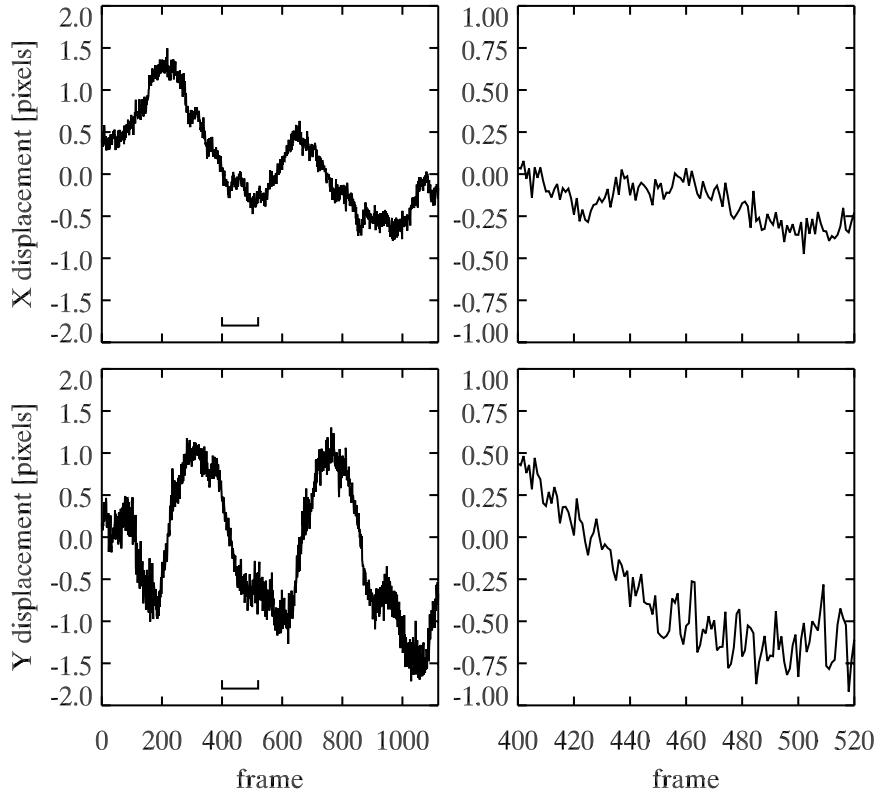

Fig. 1. Corrections for residual image displacements for the 1600- $\AA$ sequence, in the solar $X$ (upper panels) and $Y$ directions (lower panels), plotted against frame number. The enlargements in the righthand panels are for the short segments specified by the bars at left.

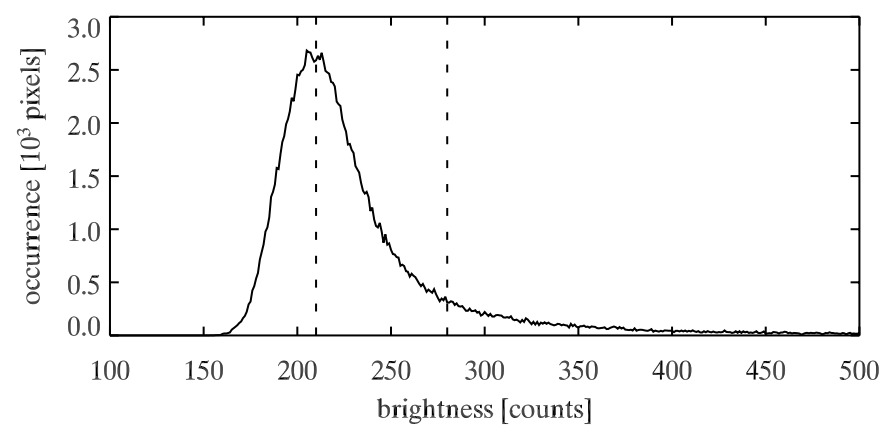

Fig. 2. Brightness histogram of one 80-min average of the $1600-\AA$ sequence. The dotted lines define the split between network (right), intermediate (middle), and internetwork (left).

corresponding coarsely aligned 1600- $\AA$ image taken $5.574 \mathrm{~s}$ before. We then applied spatial smoothing through $5 \times 5$ pixel boxcar averaging to every image, and, merging the two sequences, applied temporal smoothing per pixel by an eighteenimage boxcar average. Alignment of each individual image of the de-rotated sequences to this smoothed average yields displacement vectors per image. SolarSoft routine tr_get_disp was employed in all alignment computations.

Figure 1 shows the resulting displacement corrections for the 1600- $\AA$ sequence. These are the frame-by-frame residuals after the initial correction for differential rotation. They primarily describe pointing errors. Both $X$ and $Y$ components show oscillatory behavior with about 1.5-pixel amplitude and approximately 100-min periodicity caused by the spacecraft's orbital motion. The enlargements at right show ragged excursions with quarter-pixel amplitudes on short timescales which reflect pointing jitter. Solar rotation causes a much larger additional drift in the horizontal direction. The de-rotation correction ranges from -73.33 pixels at the equator to -72.83 pixels 

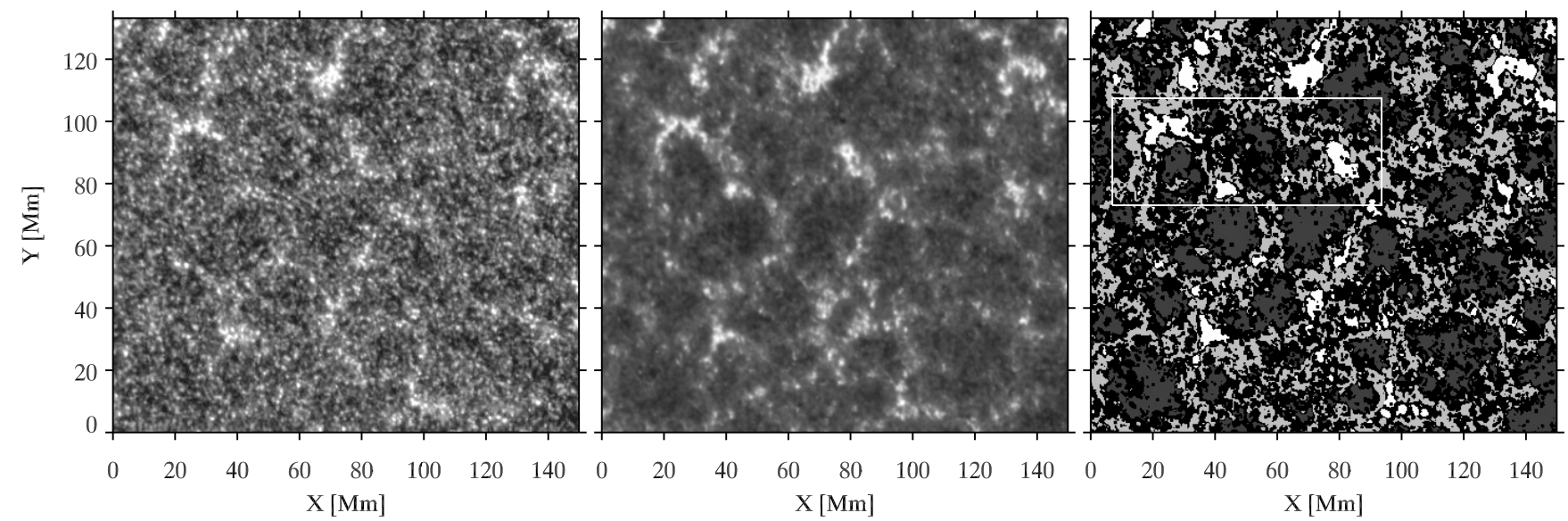

Fig. 3. First panel: sample image from the $1600-\AA$ sequence taken at 11:32:22 UT. The intensity was clipped and scaled logarithmically in order to gain contrast in the internetwork. Second panel: 80 -min 1600- $\AA$ average (12:44-14:05 UT) using the same gray scale. Third panel: the pixel masks applied to all images in Sect. 3.2. Dark gray, light gray, and white respectively denote internetwork (28 413 pixels), intermediate (36 914 pixels), and network (7430 pixels). Black pixels are discarded. The box specifies the subfield selected for the power maps in Fig. 5.

at the bottom of the field of view. Our use of whole-field alignment automatically corrects for any departures from the initially applied rotation law except for those in differential shear. The error estimates of Howard et al. (1990) suggest that the remaining shear errors are within 0.004 pixel over our range in solar $Y$ and time.

In the final step of the alignment procedure, the sophisticated algorithm described by DeForest (2004) is used to re-sample the original images onto a $432 \times 384$ pixel grid corrected for differential solar rotation, for spacecraft orbital motion and jitter, and for the re-mapping from planar to spherical coordinates. The area of incomplete sampling due to solar rotation is discarded, as is a vertical strip at the left-hand edge of the field of view which erroneously appears bright in one $1700-\AA$ image. The resulting images consist of $432 \times 3840.348 \mathrm{Mm}-$ square pixels.

For part of our analysis, i.e., the spatially averaged Fourier spectra presented in Fig. 7 in Sect. 3.2, we followed the procedure of Paper I to divide the field of view into internetwork, intermediate, and network areas through classification of the time-averaged 1600- $\AA$ brightness per pixel. Temporal averaging increases the contrast between the rapidly changing internetwork brightness and the more stable network emission. The $1600-\AA$ sequence was split into three parts of approximately 80 -min duration. Figure 2 displays the brightness distribution after averaging over one 80-min part. It has a Gaussian peak and an extended high-brightness tail. A pixel is classified as internetwork if in all three 80 -min averages its brightness remains below the left-hand dotted line, which is chosen near the three peaks. Pixels with brightness above the right-hand dotted cutoff in all three averages are classified as network. Pixels that fall between the two lines in all three averages are classified as intermediate. Pixels that change category between averages are discarded. This category amounts to $56 \%$ of all pixels due to the long sequence duration. It is large to avoid any mixing of internetwork, intermediate, and network behavior.

Particle hits were not corrected by interpolation but were removed on the basis of their high-frequency signature for the analysis in Sect. 3.2. Their single-image appearance produces anomalously strong high-frequency power. We applied a spatial mask to remove all pixels as well as their immediate neighbors that show Fourier power in excess of three times the average in the highest 50 frequency bins $(36.7-38.5 \mathrm{mHz})$.

Figure 3 presents a sample $1600-\AA$ image, one of the three 80-min averages, and the three masks.

\section{Analysis and results}

Fourier power, coherence and cross-power spectra were computed per pixel in the $1600-\AA$ and $1700-\AA$ image sequences over their full 243-min duration following the recipes in Sect. 3 of Paper I. We follow Paper I also in the presentation of the resulting power maps, spatially-averaged temporal Fourier spectra, and two-dimensional $\left(k_{\mathrm{h}}, f\right)$ diagrams. Here, the emphasis is on high-frequency behavior and its significance.

\subsection{Spatially resolved Fourier power maps}

As in Paper I we distinguish three different normalization choices in displaying Fourier power per pixel as spatially resolved maps, namely plotting the non-normalized oscillatory energy itself ("power"),

$P_{E}(x, y, f)=|\mathcal{I}(x, y, f)|^{2}$,

the fractional modulation signal obtained by dividing the oscillatory energy by the zero-frequency power ("modulation"),

$P_{f}(x, y, f)=\frac{|\mathcal{I}(x, y, f)|^{2}}{|\mathcal{I}(x, y, 0)|^{2}}$

and "Leahy" normalization obtained by dividing the energy by the zero-frequency amplitude,

$P_{\mathrm{L}}(x, y, f)=\frac{|\mathcal{I}(x, y, f)|^{2}}{|\mathcal{I}(x, y, 0)|}$

where $x$ and $y$ are spatial coordinates, $f$ is the temporal frequency, and $\mathcal{I}(x, y, f)$ denotes the Fourier transform of the intensity measured by TRACE at location $(x, y)$ at frequency $f$. 

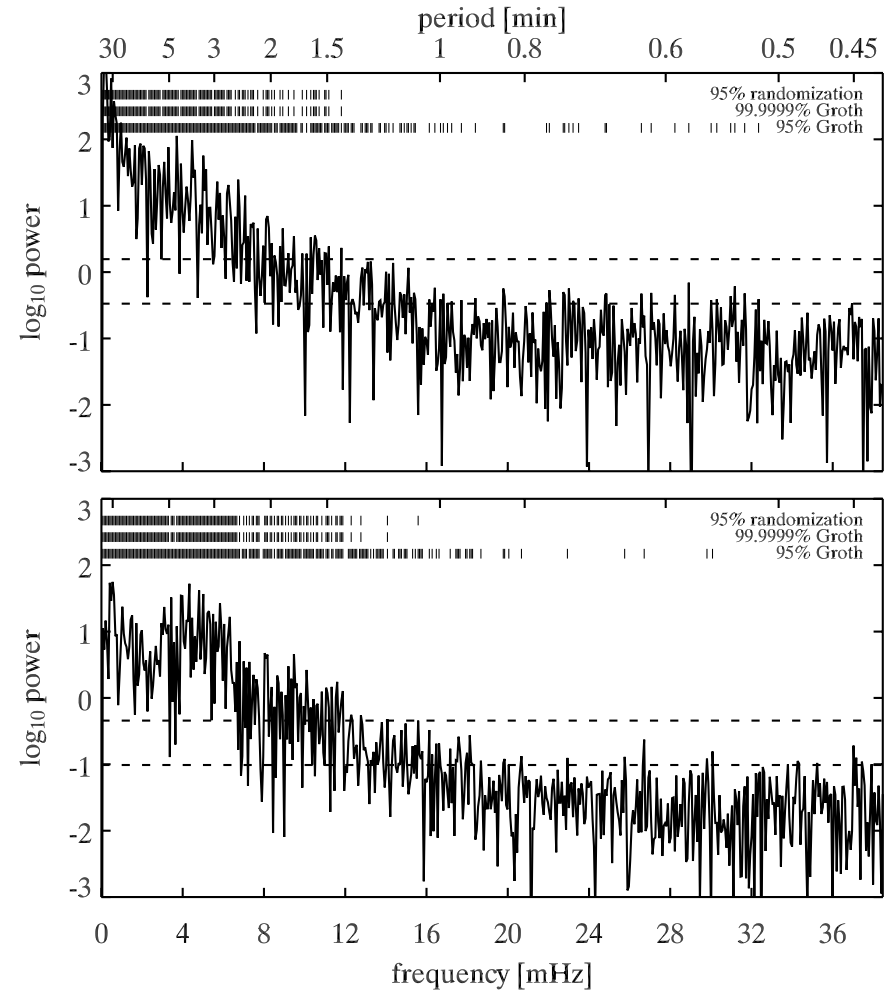

Fig. 4. Comparison of the randomization test to significance estimation following Groth (1975). Upper panel: network pixel. Lower panel: internetwork pixel. The ragged curves are the temporal Fourier power at $1600 \AA$ using TRACE data units divided by the exposure time, on logarithmic scales. In each panel, the top row of tick marks identifies all significant peaks according to the randomization test. The second and third rows identify significant peaks at the $99.9999 \%$ and 95\% significance levels using Groth's test assuming white noise and absence of signal above $f=24 \mathrm{mHz}$. The dotted lines show the corresponding cutoff levels.

Leahy normalization is used in the literature to estimate powerpeak significance (e.g., Leahy et al. 1983; Doyle et al. 1999) but was not used in Paper I.

We here add $95 \%$ significance estimation following Groth (1975) and first compare this to Fisher's method of randomization described by, e.g., Bradley (1968) and Linnell Nemec \& Nemec (1985) and applied to solar data by, e.g., O'Shea et al. (2001) and Muglach (2003). Its assumption is that there is no signal at any frequency, so that the temporal order in which the data were taken becomes irrelevant. Comparison of the actual power spectrum to the spectra of temporal permutations of the data sequence then yields a significance estimate. The test is used iteratively, progressively deleting significant peaks until no new peaks are found. It puts no constraint on the noise power distribution at any given frequency, but the assumption that all samples are temporally uncorrelated implies frequencyindependent white noise. For large data sets it becomes impractical to repeatedly compute all possible permutations. Actual tests are therefore usually limited to a few hundred permutations, but even then remain computationally expensive.

The much simpler significance estimation of Groth (1975) assumes that at any frequency the real and imaginary parts of the Fourier power have independent normal distributions.
It requires explicit specification of the noise power as a function of frequency, i.e., the noise is not assumed to be white.

We compare the randomization test with Groth's test in Fig. 4 for an internetwork and a network pixel, adopting 95\% confidence levels in both tests. In the randomization test, a power peak that is above the maximum power of the randomized data in more than $95 \%$ of 500 permutations is considered statistically significant. Such peaks are subsequently removed in the iterative re-application of this procedure until no more significant peaks are found. For Groth's test we decided from visual inspection to assume that the power spectra display white noise above $f=24 \mathrm{mHz}$. The corresponding $95 \%$ significance cutoff is 2.996 times higher than this noise level.

The top and bottom rows of tick marks in Fig. 4 specify the positions of the peaks that are estimated to be significant by the two methods. It is obvious that the randomization test is far more rigorous than Groth's test, as pointed out by Muglach (2003). The middle rows of ticks result when the upper dotted line is used as Groth-test cutoff level, at fourteen times the noise level corresponding to $99.9999 \%$ confidence. It closely matches the peak-finding by the randomization test. Thus, for these data a stringent Groth test may replace the randomization test at much smaller computational cost. This is likely to hold for other data with white noise.

The network pixel in the upper panel of Fig. 4 has larger low-frequency signal than the internetwork pixel in the lower panel, a different power hump around 5-min periodicity, and higher high-frequency noise but rather similar peak survival above the lenient $95 \%$ Groth cutoff estimate.

Figure 5 expands such comparison of internetwork versus network by displaying spatial power maps for the small but illustrative subfield specified by the rectangle in Fig. 3 for both the $1600-\AA$ and $1700-\AA$ passbands. The subfield is shown in different temporal frequency bands, with the three different normalizations (power, Leahy, and modulation, respectively), and finally also without normalization but with all pixels having power below the $95 \%$ Groth cutoff made black.

The first column shows low-frequency power. It shows the stable nature of bright network. The power-normalized modulation maps show noisy behavior from the division because the frequency band is close to zero $\mathrm{Hz}$.

The second-column frequency range of $2.6-3.6 \mathrm{mHz}$ corresponds to periodicities around $5 \mathrm{~min}$. The network appears power-bright in the unnormalized maps, about equal to the internetwork in the amplitude-normalized Leahy maps, and power-dark in the modulation maps. Thus, the choice of normalization affects the apparent relative dominance of network and internetwork oscillations, as discussed extensively in Paper I. Note that in all representations a power-dark moat appears around the network.

The 5-7 $\mathrm{mHz}$ maps describe the chromospheric threeminute oscillation which pervades internetwork areas (e.g., Rutten 1995). They indeed show the network power-dark in all representations. There are irregular power-bright "aureole" patches near network (cf. Paper I).

The two high-frequency columns on which we concentrate here illustrate the care that must be taken in interpreting such power maps. In the rightmost column $(28-32 \mathrm{mHz})$ the 


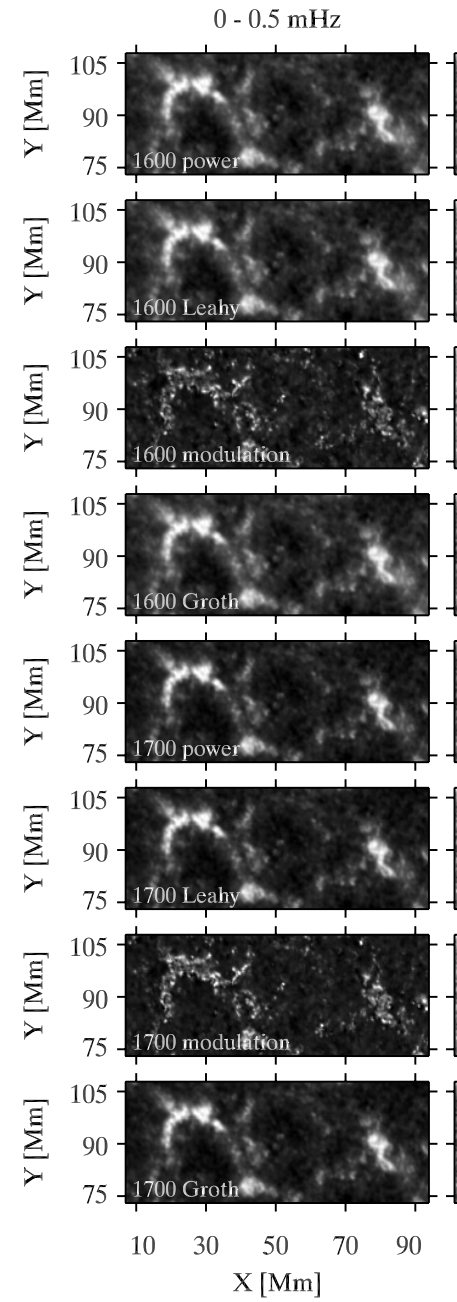

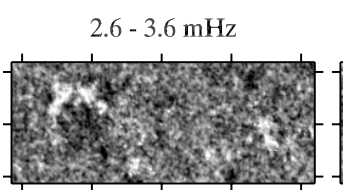
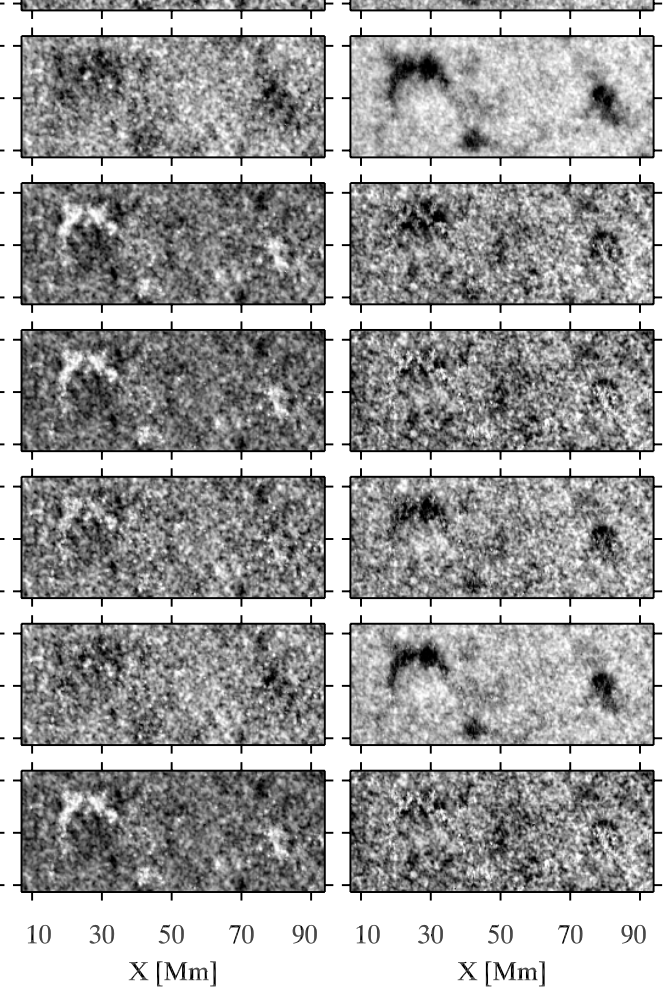

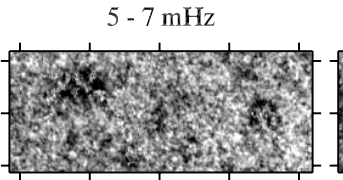

$12-16 \mathrm{mHz}$
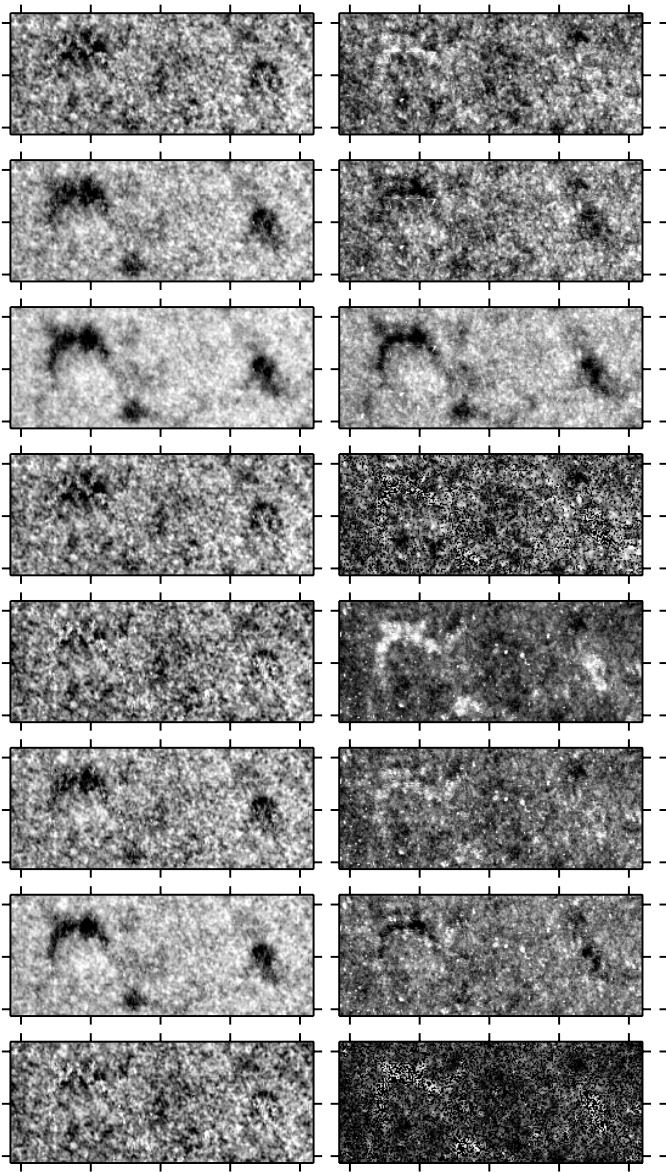
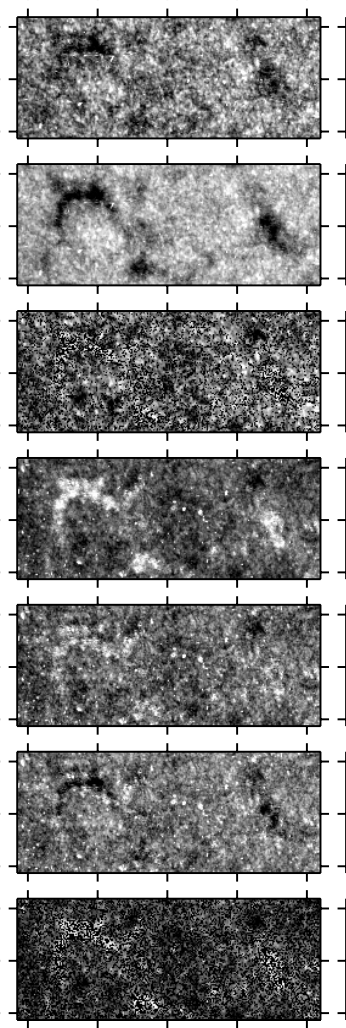

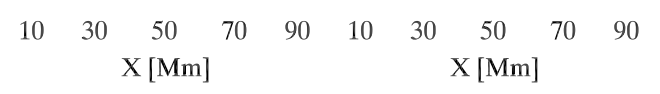
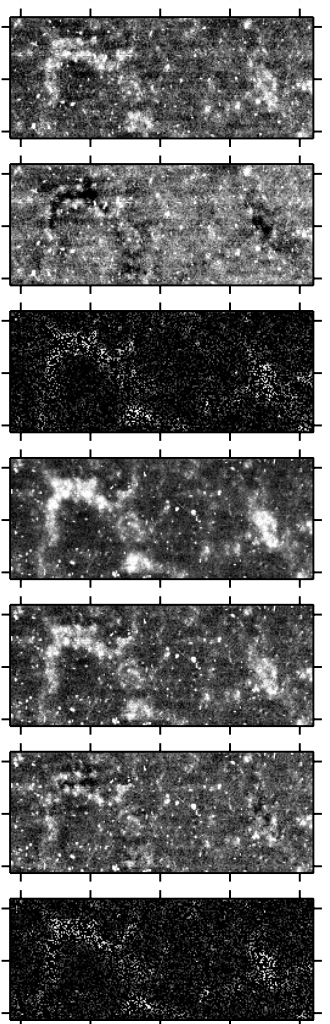

$28-32 \mathrm{mHz}$
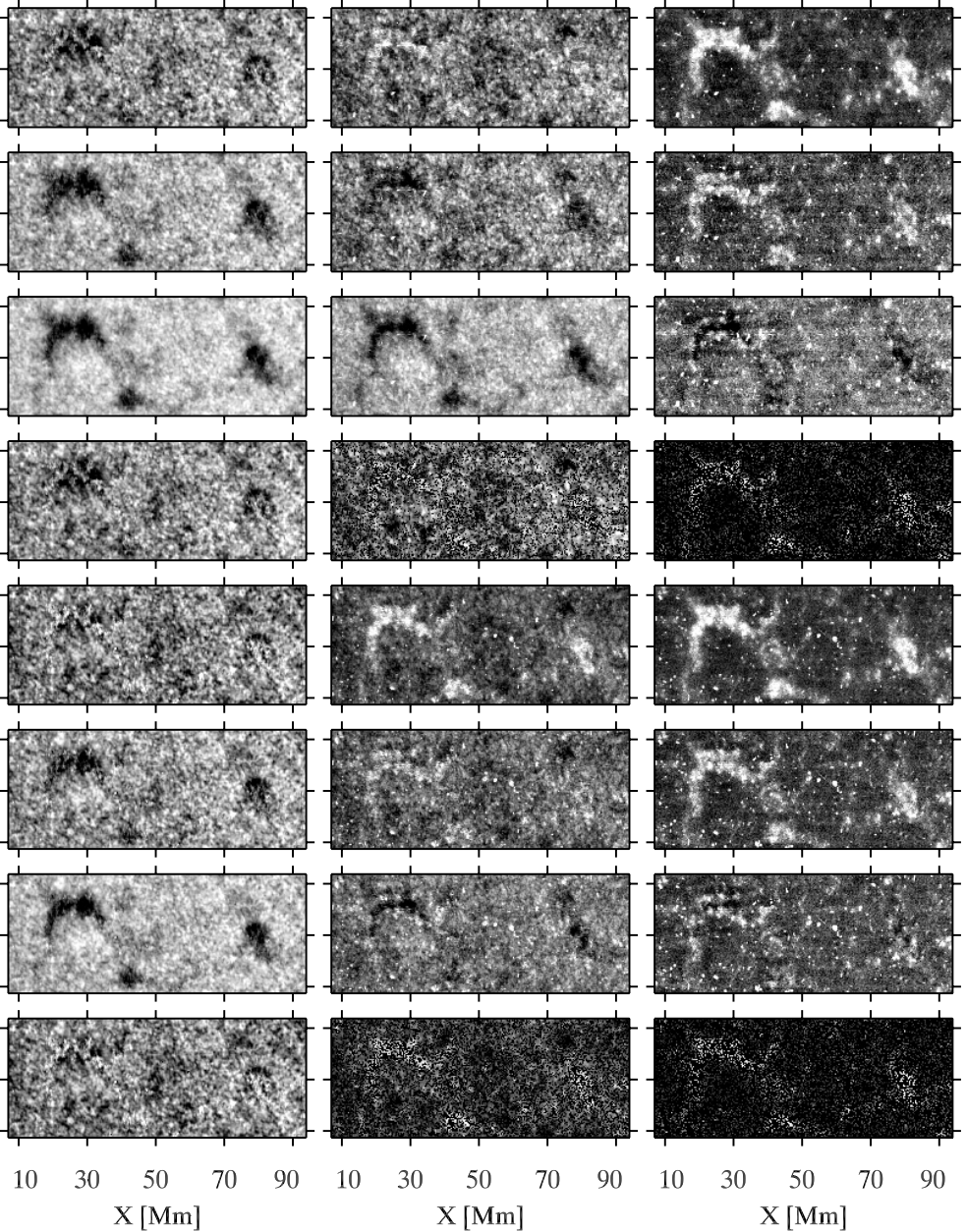

Fig. 5. Spatially resolved power maps using different methods of normalization for the subfield shown in Fig. 3. The grayscale displays the logarithm of the temporal Fourier power, clipped to improve contrast. Columns: different frequency bands as specified at the top. Rows: $1600-\AA$ and $1700-\AA$ passbands, with different power normalization as specified in the first column.
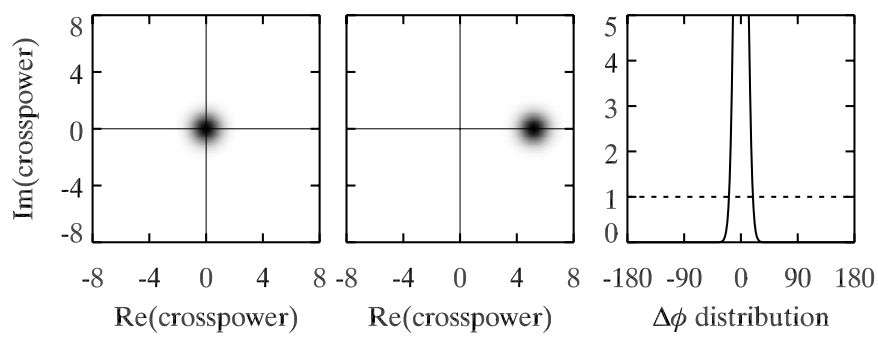

Fig. 6. Illustration of vector-averaging phase differences for 30000 pixels. First panel: distribution of the cross-power vector sum for pure Gaussian noise in the complex plane. Second panel: same as the first panel, but with a signal with amplitude of only $3 \%$ of the rms noise with 0 degree phase difference added. The vector summation of the 30000 samples shifts the scattercloud significantly to the right. Third panel: corresponding phase-difference distributions for pure noise (dashed, flat) and with the signal added (solid, peaked). The latter reaches 18.4 at phase difference 0 with 20-degree half-width.

network stands out very brightly in the unnormalized power maps, inviting a claim that the magnetic elements making up the network display high-frequency wave heating. On the other hand, the network appears power-dark in the modulation maps, inviting a claim that high-frequency oscillations are suppressed in magnetic elements. However, the close spatial correspondence of both these bright and dark features with the bright network in the unnormalized maps in the first column suggests strongly that they are simply due to the larger overall network brightness. A similar power-contrast flip is seen in the $12-16 \mathrm{mHz}$ maps for the $1700-\AA$ passband, but the unnormalized 1600- $\AA$ map for these frequencies appears rather featureless. The latter copies directly into the Groth map, but with considerable pixel deletion wherever the power averaged over the frequency range drops below the cutoff. In the rightmost column the Groth maps accept only a minor fraction of the pixels as significant, both for the network and the internetwork. A more strict criterion, and certainly the $95 \%$ iterative randomization test, would reject all. The patterns seen in the other $28-32 \mathrm{mHz}$ maps thus are most likely artifacts caused by sources of high-frequency errors with some sensitivity to the low-frequency power. Note that Leahy normalization diminishes the apparent structure for $1700 \AA$ but turns it power-dark at $1600 \AA$. 


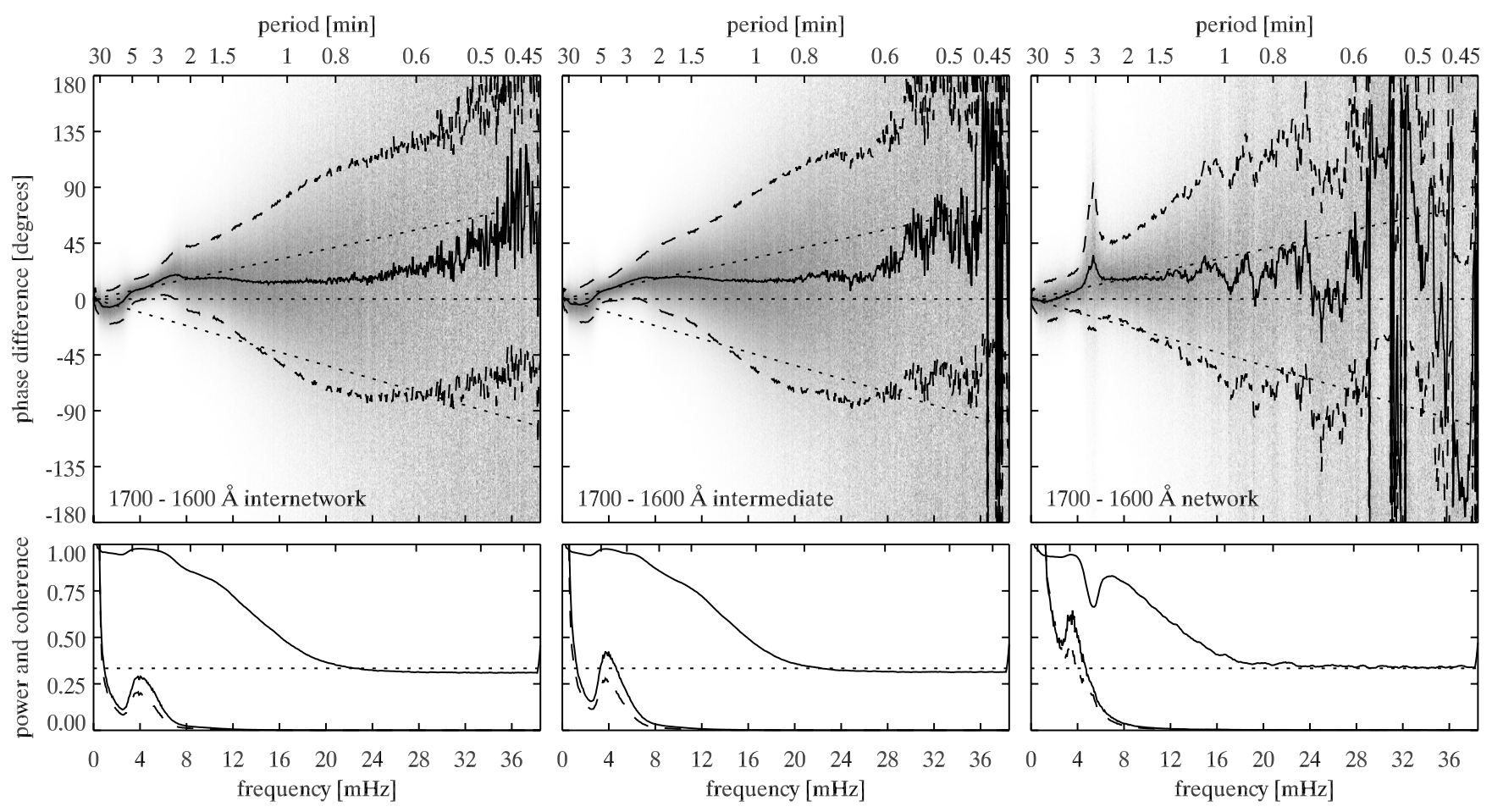

Fig. 7. Temporal Fourier spectra, spatially averaged over internetwork (left), intermediate (middle), and network (right), plotted against frequency up to the Nyquist limit with the corresponding periodicities shown along the top. The format corresponds to Figs. 18-19 of Paper I, adding dotted lines indicating the sampling time delays and omitting $1 \sigma \mathrm{rms}$ estimates for power and coherency to avoid clutter. Upper panels: phase-difference spectra. Lower panels: coherence (upper curves) and power spectra (solid for $1600 \AA$, dashed for $1700 \AA$ ). The random-noise estimate for the coherence is $C=0.33$. The power spectra are on linear scales and are all scaled by the same factor.

The bright specks in the $28-32 \mathrm{mHz}$ maps are due to particle hits. They produce high-frequency signal through their instantaneous appearance. The pattern of horizontal stripes results from TRACE's JPEG data compression (K. Muglach, private communication). It appears as a grid pattern with 8-pixel mesh size in comparable power maps computed from the original non-aligned image sequences. The compensation for solar rotation smears out the vertical grid components, leaving only the horizontal ones.

\subsection{Spatially averaged Fourier power, phase difference and coherence}

We now turn to temporal Fourier analysis with spatial averaging over the different pixel categories defined by the third panel of Fig. 3. The averaging is performed on the Fourier measurements per pixel. It reduces the noise in these measurements and therefore improves the detection of relatively small modulation signals. Following Paper I, the power and coherence are averaged directly over all relevant pixels. In the present analysis we compute coherence per pixel using frequency smoothing over 9 bins rather than 5 .

The phase differences are again averaged with cross-power weighting as introduced by Lites \& Chipman (1979), i.e., the spatial average over the phase differences of all pixels transmitted by the mask per frequency bin is defined as the angle of the vector sum of the cross-powers of all contributing pixels with reference to the real axis. The advantage of such vector averaging is that it makes signals stand out even in the presence of much larger noise. For pure noise the vector mean does not go to zero or some other definite value but fluctuates randomly over the full -180 to +180 degree range between adjacent frequency bins. A small signal, much smaller than the noise, may therefore emerge as a systematic pattern across multiple bins.

This is illustrated by Fig. 6 which displays simulation results for vector-averaged cross-power distributions of pure noise (first panel) and of pure noise with a much smaller superimposed signal with fixed phase difference (second panel). In the latter case, the vector addition over 30000 pixels with small but systematic signal shifts the much wider scattercloud to a location well separated from the origin, making the phasedifference distribution in the third panel strongly peaked.

Figure 7 presents results from the new TRACE sequences in the form of power, coherence, and phase-difference spectra with spatial averaging separated between the internetwork, intermediate, and network areas. The format is similar to Figs. 18-22 of Paper I but adds two dotted lines in the phasedifference panels. These are the phase shifts associated with the temporal sampling delay due to the non-simultaneous exposures in the two passbands. We have aligned the two sequences to match the closest pair combinations, corresponding to the upper dotted lines. All of our phase-difference evaluations are corrected for this sampling offset, which means that signals that are intrinsically in phase in the two passbands should indeed end up along the horizontal $\Delta \phi=0$ axis. Measurements that end up on a dotted line imply modulation with phase 

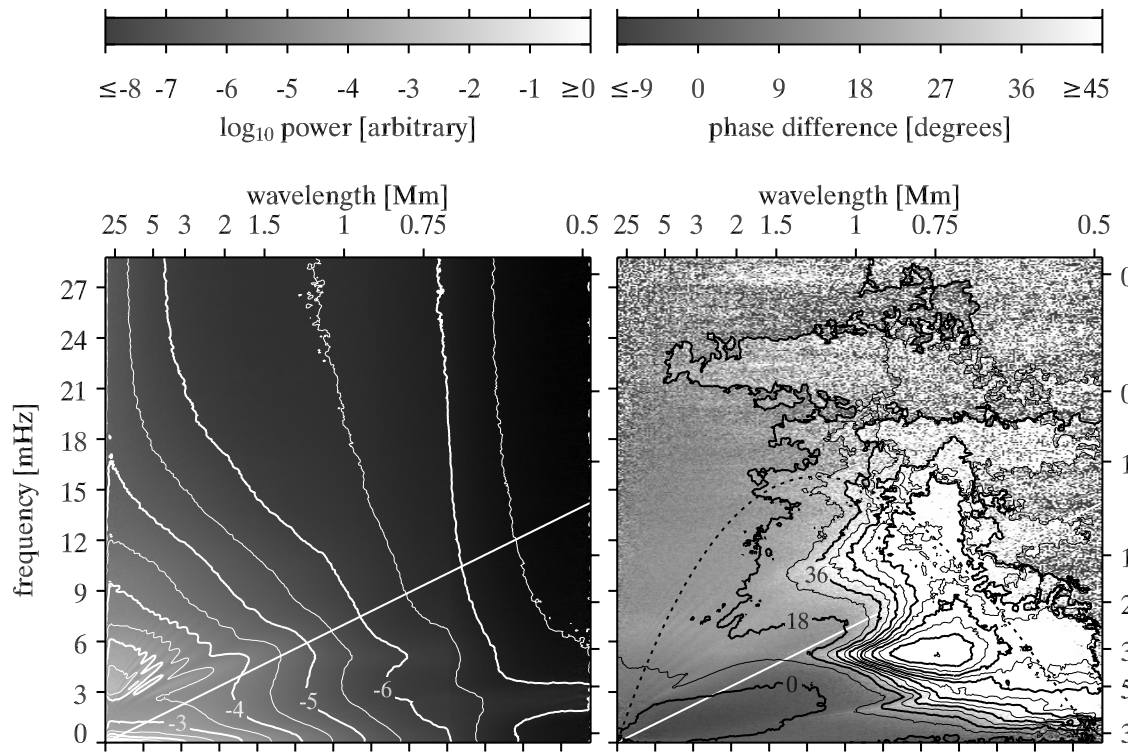

$\begin{array}{lllllllllllll}0 & 1 & 2 & 3 & 4 & 5 & 6 & 7 & 8 & 9 & 10 & 11 & 12\end{array}$ horizontal wave number $\left[\mathrm{Mm}^{-1}\right]$ $\begin{array}{lllllll}0.5 & 25 & 5 & 3 & 2 & 1.5 & \text { wavelength [Mm] }\end{array}$

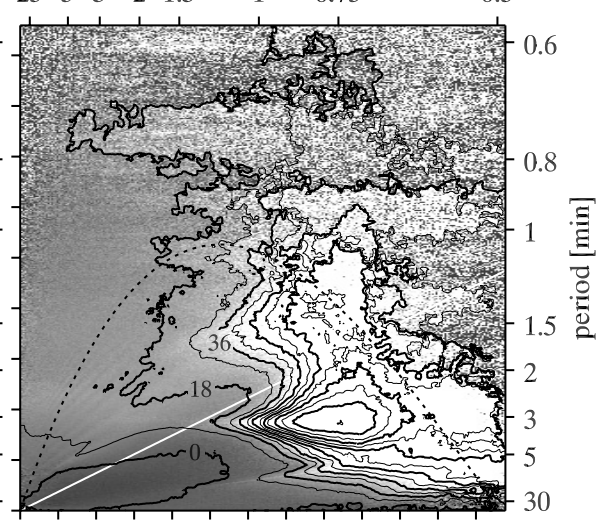

$\begin{array}{lllllllllllll}0 & 1 & 2 & 3 & 4 & 5 & 6 & 7 & 8 & 9 & 10 & 11 & 12\end{array}$ horizontal wave number $\left[\mathrm{Mm}^{-1}\right]$
Fig. 8. Left: power in the $1600-\AA$ image sequence plotted as function of horizontal wave number $k_{\mathrm{h}}$ and temporal frequency $f$. The logarithmic grayscale is clipped to show the ridges around $1 \mathrm{Mm}^{-1}$ and $5 \mathrm{mHz}$. The slanted line is the Lamb line $f=$ $(1 / 2 \pi) c_{\mathrm{s}} k_{\mathrm{h}}$ with $c_{\mathrm{s}}=7 \mathrm{~km} \mathrm{~s}^{-1}$. Right: corresponding phase differences between the $1700-\AA$ and $1600-\AA$ image sequences. To avoid figure clutter, contours are only shown if they lie below the dashed curve or enclose a large area. The grayscale is clipped at -9 and 45 degrees to increase contrast. The white blob peaking at $k_{\mathrm{h}}=9 \mathrm{Mm}^{-1}$ and $f=5 \mathrm{mHz}$ reaches 110 -degree difference. The pepper-and-salt regions reflect noise. delay exactly matching the corresponding sampling delay. The grayscaled scattercloud represents individual pixels. In the case of pure noise the $1 \sigma \mathrm{rms}$ estimates cover $68 \%$ of the full figure height around a randomly fluctuating mean.

The results in Fig. 7 are similar to those in Fig. 18 of Paper I except for the high-frequency phase differences of interest here. The present results are more reliable thanks to the regular sampling cadences, lower data compression, and better image alignment.

The internetwork phase differences reach a wide maximum at $f \approx 7 \mathrm{mHz}$ and then remain well defined at positive values up to the Nyquist frequency, but with increasing noise above $20 \mathrm{mHz}$. There is no drop to negative values as in Paper I, which we now attribute to the cross-alignment used there as discussed in Sect. 2 above. However, the present results also show a drift to the phase difference associated with the timing delay at high frequencies. The internetwork power spectra show acoustic humps around $4 \mathrm{~min}$ and become negligible above $12 \mathrm{mHz}$. The coherence also peaks around $4 \mathrm{mHz}$ and drops to the 9-bin noise level near $20 \mathrm{mHz}$.

The network phase differences in the third column of Fig. 7 are much noisier due to the far smaller number of pixels. Nevertheless, they show a narrow peak of increased phase difference and reduced coherence around three-minute periodicity which is not present in Fig. 18 of Paper I and which we deem significant. At high frequencies they become more erratic and shift to the timing correction line, which points to a systematic error.

The intermediate-class pixels in the center column of Fig. 7 produce primarily internetwork-like behavior.

\subsection{Two-dimensional Fourier power and phase difference}

Figure 8 presents two-dimensional Fourier power and phase difference spectra in the form of $\left(k_{\mathrm{h}}, f\right)$ diagrams. They mix the network, internetwork and intermediate areas. Particle hits were not removed because their contribution to the noise is small except at high spatial and temporal frequencies where the diagrams are noisy anyhow.

The power and phase differences are averaged over rings of constant $k_{\mathrm{h}}$, with $k_{\mathrm{h}}^{2}=k_{x}^{2}+k_{y}^{2}$, assuming absence of preferred horizontal propagation directions. The number of samples per ring increases with $k_{\mathrm{h}}$ up to the Nyquist frequency per axis $k_{x, \mathrm{Ny}}=k_{y, \mathrm{Ny}}=9.0 \mathrm{Mm}^{-1}$. Beyond this value, $k_{\mathrm{h}}$ can still be computed but with fewer samples and increasing loss of isotropy in each successive bin, up to $k_{\mathrm{h}}=\sqrt{2} k_{x, \mathrm{Ny}}=$ $12.8 \mathrm{Mm}^{-1}$ which samples only oblique propagation.

The left-hand panel of Fig. 8 shows the $\left(k_{\mathrm{h}}, f\right)$ diagram for 1600 - $\AA$ power. The acoustic $p$-mode ridges and pseudo-ridges above the Lamb line were extensively discussed in Paper I. There is no particular structure evident in the high-frequency regime of interest here. At low frequencies there is a ridge of enhanced power at high spatial wavenumbers, approximately corresponding to $f=(1 / 2 \pi) 2 \mathrm{~km} \mathrm{~s}^{-1}$ which is caused by the compensation for solar rotation. Features that are fixed to the CCD camera, such as the results of an imperfect flat field, or "hot" pixels, move apparently with this speed against the direction of solar rotation and produce power.

The right-hand panel of Fig. 8 displays the corresponding $\left(k_{\mathrm{h}}, f\right)$ diagram for phase difference between the $1700-\AA$ and 1600 - $\AA$ sequences. The acoustic ridges stand out through larger phase difference, as discussed in Paper I. The wedge of negative phase difference at low frequencies and wave numbers was attributed to atmospheric gravity waves by Rutten \& Krijger (2003). The effects of solar rotation are again visible as a ridge of slightly increased phase difference. We attribute this increase to the systematic $(-0.285,0.273)$-pixel image offset between the two passbands before alignment, which causes apparently traveling features fixed to the CCD to appear at a given solar location with some time delay in the two passbands. 
At the highest temporal frequencies of interest here, noise is easily identified as pepper-and-salt patterning where the phase differences jump widely from one bin to the next. A large patch of smooth variation extends up to about $20 \mathrm{mHz}$ in frequency and $4 \mathrm{Mm}^{-1}$ in wavenumber. This patch contributes most to the definite phase behavior in Fig. 7, which is therefore set by these spatial scales.

The conspicuous white blob of large positive phase difference located at $0.75 \mathrm{Mm}$ wavelength and 3-min periodicity, with an extended tail upward, is enigmatic. The left-hand diagram suggests enhanced power at this location. It seems likely that the peak in the network panel of Fig. 7 corresponds to this blob, and that therefore the source should be sought in the network. It is very tempting to attribute it to solar three-minute waves with small horizontal extent in magnetic elements. Its exceedingly large value (up to 110-degree difference at its center), the clear reduction in coherence, and the lack of such a blob in comparable phase-difference diagrams sampling the $\mathrm{Ca}$ II $\mathrm{H}$ line core and inner wing from observations made with the Dutch Open Telescope (unpublished analysis analogous to Fig. 7 of Rutten et al. 2004), would then suggest phenomena in the transition region that leave a signature in these data through the C IV doublet at $\lambda=1548 \AA$ and $1550 \AA$ (cf. Handy et al. 1998). However, the blob lies at the spatial Nyquist frequency per horizontal axis, and its shape varies with changes in the image alignment procedure. We reluctantly conclude that the blob is likely a TRACE artifact, presumably of instrumental origin, introduced by the data processing, or a combination of these.

\section{Discussion}

We find intriguing high-frequency behavior in all our Fourier displays, but at the same time also find reasons to disbelieve these signatures above $20 \mathrm{mHz}$ or even lower frequencies. The pixel-by-pixel power maps in Fig. 5 have disconcerting high-frequency contrast sensitivity to the type of normalization above $10 \mathrm{mHz}$. The spatial averaging in Figs. 7 and 8, respectively over mask-selected pixel types and annuli, improves the significance in phase-difference measurement, but the highfrequency behavior in Fig. 7 is puzzling in the trends towards the timing delay lines and the absence of purely random behavior even at the highest frequencies. The prominent white blob in the phase-difference panel of Fig. 8 is presumably an artifact.

The phase-difference averaging with cross-power weighting over the different pixel categories employed in Sect. 3.2 is the most sensitive method to identify weak oscillation signatures in the presence of noise. Each phase-difference diagram in Fig. 7 indicates systematic non-random behavior out to frequencies far beyond the extent of measurable power or even of measurable coherence. This was already the case in Figs. 18 and 19 of Paper I and also in the similar diagrams from groundbased Ca II H spectrometry in Figs. 20-22 of Paper I. It is attractive to believe that the cross-power weighting indeed enhances the sensitivity of the phase-difference measurement to very small signals otherwise drowned in noise out to well above the coherence limit of at most $20 \mathrm{mHz}$, but it is alarming that even at the highest frequencies the phase differences do not show the randomness expected, and that in all three panels in
Fig. 7 it seems to favor the instrumental timing correction. It is likely that residual image alignment errors are the cause of this anomalous behavior.

It is well-known that the increasing lack of response due to wide contribution functions (e.g., Beckers \& Milkey 1975; Deubner 1976; Schmieder \& Mein 1980; Durrant 1980) hampers the detection of high-frequency signals. It was recently elaborated in TRACE context by Fossum \& Carlsson (2004). In addition, we have learned from M. Carlsson (private communication) that simulations of acoustic waves propagating upward in the solar atmosphere as in the well-known Ca II $\mathrm{H}_{2 \mathrm{~V}}$ grain simulation of Carlsson \& Stein (1997) subjected to computational 1700-1600-A phase-difference analysis which emulates the observational analysis presented here meets unexpected computational problems at low signal to noise and high frequencies.

On the other hand, we have reproduced our phasedifference results in tests using double precision computation. Very similar non-random positive phase-difference behavior also appears up to $20 \mathrm{mHz}$ in Fig. 20 of Paper I, based on the Ca II H slit spectrometry of Lites et al. (1993) and measured from Ca II H wing intensities and Fe I blend Doppler shifts formed at lower and similar heights as the ultraviolet continua sampled by TRACE. The comparable signature in $I-I$ and $V-V$ diagnostics with negative $V-I$ lag shown there is in agreement with acoustic waves. The steep $V-V$ signature of upward propagation in internetwork areas present in the lowerleft panel of Fig. 21 of Paper I seems significant also.

In summary, the coherence spectra in Fig. 7, the close agreement of the phase differences in that figure with those from Ca II H spectrometry in Paper I, and the smoothness of the corresponding gray area in Fig. 8, all taken together lead us to believe that the phase-difference signals derived from these new TRACE sequences have a solar origin up to $20 \mathrm{mHz}$ at least in the internetwork, and are to be attributed to acoustic waves.

This conclusion supports the detection of high-frequency acoustic waves by Wunnenberg et al. (2002) as significant Doppler-shift power in the $10-20 \mathrm{mHz}$ frequency band from differential Fe I measurements addressing similar atmospheric heights. The drop of power with frequency in our Figs. 4, 7 and 8 suggests that their detection is dominated by the lower frequencies in this band. Our results indicate wave presence also at the higher frequencies.

The ultraviolet continua used here suffer from strong scattering while the TRACE filter bandwidths are wide and overlap considerably. Numerical simulations as those presently underway at Oslo may explain how and why the phase differences in Figs. 7 and 8 level out at positive values. In our opinion, comparison with detailed numerical simulations is also required to substantiate any claim that acoustic waves in the $10-20 \mathrm{mHz}$ regime compensate fully for the radiative losses of the chromosphere.

\section{Conclusion}

New ultraviolet image sequences from TRACE give evidence of brightness modulation up to $20 \mathrm{mHz}$ in quiet-sun 
internetwork. We interpret this signal as a signature of acoustic waves. It is similarly present in $\mathrm{Ca}$ II $\mathrm{H}$ and Fe I $I-I$ and $V-V$ phase-difference spectra in Fig. 20 of Paper I and it supports the detection of acoustic wave power in the $10-20 \mathrm{mHz}$ frequency band from Fe I Doppler-shift measurements by Wunnenberg et al. (2002). The evidence for modulation at higher frequencies remains inconclusive.

TRACE-like ultraviolet imaging will be achieved with the Atmospheric Imaging Assembly on NASA's Solar Dynamics Observatory, but it is not yet clear whether its hardware and operation will permit better high-frequency modulation measurement than with TRACE. New ground-based telescope technology, in particular large aperture combined with adaptive optics, will provide accurate Doppler shifts from integral field spectroscopy at high cadence and low noise of the same layers using appropriate spectral lines in the optical. Numerical simulations may contribute quantification of the corresponding energy budgets.

Acknowledgements. We thank M. Carlsson for suggesting these TRACE observations to the third author and for sharing simulation insights in the intricacies of phase-difference determination with the second author. We also thank C. E. DeForest, J. Leenaarts, C. C. Kankelborg, J. M. Krijger, B. W. Lites, K. Muglach and R. A. Shine for advice and discussions, and the referee for suggesting many clarifications. A. G. de Wijn and R. J. Rutten acknowledge travel support from NASA (contract NAS5-38099) and the Leids Kerkhoven-Bosscha Fonds, and are indebted to the Lockheed Martin Solar and Astrophysics Lab. at Palo Alto, the solar physics group of Montana State University at Bozeman, and the High Altitude Observatory at Boulder for hospitality.

\section{References}

Beckers, J. M., \& Milkey, R. W. 1975, Sol. Phys., 43, 289

Biermann, L. 1948, ZA, 25, 161
Bradley, J. V. 1968, Distribution-free statistical tests (Prentice-Hall) Carlsson, M., \& Stein, R. F. 1997, ApJ, 481, 500

DeForest, C. E. 2004, Sol. Phys., 219, 3

Deubner, F.-L. 1976, A\&A, 51, 189

Doyle, J. G., van den Oord, G. H. J., O'Shea, E., \& Banerjee, D. 1999, A\&A, 347, 335

Durrant, C. J. 1980, A\&A, 91, 251

Fontenla, J. M., Avrett, E. H., \& Loeser, R. 1993, ApJ, 406, 319

Fossum, A., \& Carlsson, M. 2004, in Waves, Oscillations and SmallScale Transients Events in the Solar Atmosphere: Joint View from SOHO and TRACE, Proc. of SOHO 13 (Noordwijk: ESA Publ. Div., ESTEC), ESA SP-547, 125

Groth, E. J. 1975, ApJS, 29, 285

Handy, B. N., Bruner, M. E., Tarbell, T. D., et al. 1998, Sol. Phys., 183,29

Handy, B. N., Acton, L. W., Kankelborg, C. C., et al. 1999, Sol. Phys., 187,229

Howard, R. F., Harvey, J. W., \& Forgach, S. 1990, Sol. Phys., 130, 295

Krijger, J. M., Rutten, R. J., Lites, B. W., et al. 2001, A\&A, 379, 1052

Leahy, D. A., Darbro, W., Elsner, R. F., et al. 1983, ApJ, 266, 160

Linnell Nemec, A. F., \& Nemec, J. M. 1985, AJ, 90, 2317

Lites, B. W., \& Chipman, E. G. 1979, ApJ, 231, 570

Lites, B. W., Rutten, R. J., \& Kalkofen, W. 1993, ApJ, 414, 345

Muglach, K. 2003, A\&A, 401, 685

O’Shea, E., Banerjee, D., Doyle, J. G., Fleck, B., \& Murtagh, F. 2001, A\&A, 368, 1095

Rutten, R. J. 1995, in Helioseismology, ed. J. T. Hoeksema, V. Domingo, B. Fleck, \& B. Battrick, Proc. Fourth SOHO Workshop (Noordwijk: ESA Publ. Div., ESTEC), ESA SP-376 Vol. 1, 151

Rutten, R. J., \& Krijger, J. M. 2003, A\&A, 407, 735

Rutten, R. J., de Wijn, A. G., \& Sütterlin, P. 2004, A\&A, 416, 333

Schmieder, B., \& Mein, N. 1980, A\&A, 84, 99

Schwarzschild, M. 1948, ApJ, 107, 1

Wunnenberg, M., Kneer, F., \& Hirzberger, J. 2002, A\&A, 395, L51 